\title{
Research on Risk Sharing of Creative Industrial Park by Recycling Old Industrial Buildings
}

\author{
Qian $\mathrm{Wu}$ Jiayu Li Xiaoyan Ding Li Wang $\mathrm{Na} \mathrm{Li}$ \\ School of Civil Engineering, Xi'an University of Architecture and Technology \\ Shanxi, China \\ 2234279463@qq.com \\ 旧工业建筑再生利用为创意产业园的 \\ 风险分担研究 \\ 武乾 李佳昱 丁小燕 王力 李娜 \\ 西安建筑科技大学土大工程学院 \\ 陕西 710055 , 中国 \\ 2234279463@qq.com
}

\begin{abstract}
Compared with ordinary buildings, Old industrial buildings have short construction period; Lack of policies, regulations and technical standards. In order to ensure the successful implementation of the old industrial building recycling project, all participants must share the project risks reasonably. This paper takes the project of old industrial building recycling as the research object. Firstly, the Delphi method is adopted to identify the project risk. Based on the incomplete contract theory, the risk is clearly divided into the contracting stage and the risk of the performance stage. Secondly, based on participant satisfaction, build index system of risk sharing, using C-OWA operator for risk sharing weighting, avoid the ill effects of the expert subjective preferences, under TOPSIS and UT theory, form a sharing mode that is complete, reasonably define the responsibility attribution of risks and determine the optimal sharing proportion of Shared risks. Finally, the model is applied to a creative industrial park project transformed from a steel mill in Shanxi province to prove the rationality of the model, and provide reference for the formulation of risk sharing scheme of China's old industrial building recycling project.
\end{abstract}

Keywords—old industrial building; recycling; creative industry park;risk sharing

摘要一旧工业建筑再生利用后与普通建筑物相比, 具有建设周 期短, 缺乏政策法规及技术标准等特点。为保证旧工业建筑再 生利用项目成功实施, 各参与方必须合理分担项目风险。本文 以旧工业建筑再生利用为创意产业园的项目为研究对象, 首先 采用 Delphi 法识别项目风险, 依据不完全契约理论, 将风险 明确划分为缔约阶段风险与履约阶段风险; 其次构建基于参与 方满意度的风险分担指标体系, 利用 C-OWA 算子对风险分担指 标赋权, 避免专家主观偏好带来的不良影响, 在此前提下将理 想解法 (TOPSIS) 与效用理论 (UT) 相结合, 形成一套具有两者优 势及完整的风险分担模型，合理界定风险的责任归属和确定共 担风险的最优分担比例; 最后将模型应用到由陕西某钢厂改造 而成的创意产业园项目，证明该模型的合理性，为我国旧工业
建筑再生利用项目风险分担方案制定提供参考。

关键词一旧工业建筑，再生利用，创意产业园，风险分担

\section{I. 引言}

随着我国城市经济发展进入新常态, 原本地处城市 边缘的旧工业建筑逐渐占据城市中心地带。它们的闲置 不但造成了土地资源的极大浪费，更是阻碍了城市的发 展。对城市中大量闲置的旧工业建筑进行改造再利用满 足建设资源节约型社会的要求, 逐渐受到我国政府的认 同和倡导。旧工业建筑再生利用是指对旧工业建筑物进 行重新规划设计并实现其功能转型, 其过程参与方不单 一, 存在着建设周期短、建造技术不成熟、相关法律法 规不健全等各种不确定的风险因素, 如何合理划分各参 与方承担风险的责任归属与共担风险的最优分配比例是 保证项目成功的关键。

国内学者针对旧工业建筑再生利用风险管理提出了 许多不同观点。樊胜军 ${ }^{[1]}$ (2008) 利用 AHP 法 ABC 法 分类原理对旧工业厂房改造工程施工阶段的风险进行评 级, 确定风险影响大小, 采取不同的风险策略。刘文 ${ }^{[2]}$

(2012) 利用 AHP 法和风险评判矩阵将旧工业建筑改 造加固风险分为四个等级, 便于辨识风险源和量化风险 影响。高亚男 ${ }^{[3]}$ (2013)采用可拓法和改进的层次分析法对 单层工业厂房投资决策阶段的风险进行了分析, 基于评 价角度实现对旧工业建筑再利用项目风险的定量研究。

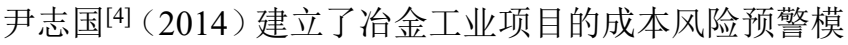
型和基于系统动力学的成本控制模型, 提出适合冶金工 业项目的成本风险管理思路与模式。余晓松 ${ }^{[5]}$ (2015) 针 对旧工业建筑改造再利用全过程风险进行评价, 采用 
ISM 分析模型对风险因素进行分级，利用主成分分析法 对风险研究进行评价, 通过实际改造项目进行可行性验 证，并对各类风险提出应对策略。

上述文献从不同角度丰富了旧工业建筑再利用的风 险管理的相关理论，但研究仍存在以下不足之处：研究 成果不断增加, 但更偏向于主观性计算和分析; 在研究 思路方面, 鲜有文献从定量角度提出风险责任归属及最 优分担比例等一套完整的风险分担机制。针对以上不足， 本文重点对旧工业建筑再生利用为创意产业园的风险分 担进行研究, 以合同为纽带将风险划分为缔约阶段风险 和履约阶段风险, 然后构建基于 TOPSIS (Technique for Order Preference by Similarity to an Ideal Solution)法的风 险初步分担模型, 界定出需要单方承担与多方共担的风 险, 并借助效用理论(UT) 模型, 确定双方满意的最优风 险分担比例, 以达到项目整体效用最大化, 为旧工业建 筑再生利用为创意产业园项目风险的合理分担提供思路。

\section{II. 旧工业建筑再生利用项目的风险识别}

风险合理分担的基础是风险识别，风险识别常用的 方法主要包括：SWOT 技术、故障树法、Delphi 法、头 脑风暴法、WBS-RBS 法等。Delphi 法相较于其他方法有 坚实的理论基础与实践基础, 对专家的选择具有较强针 对性, 使每位专家独立自主的作出判断, 对风险因素的
评判较为准确。综合考虑旧工业建筑再生利用项目的特 点, 本文采用 Delphi 法识别旧工业建筑再生利用项目风 险因素, 邀请 13 位参与旧工业建筑再生利用项目建设的 现场技术及管理人员、以及 7 位从事旧工业建筑再生利 用项目研究的大学教授等共 20 人组成专家小组对风险进 行识别。

契约是对事件相关方在责任和利益分配上的约定, 是对相关方行为界限的规定 ${ }^{[6]}$, 一般情况下, 人们不可能 预测或掌控所有未来将要发生的事情, 所以, 在实际合 作中签订的均为不完全契约合同。针对旧工业建筑再生 利用项目而言, 合同中规定了项目中参与方需要承担的 风险，这表明风险分担是合同的重要组成部分，风险分 担会随着合同内容的变化而发生改变。合同按照时间维 度可分为初始合同与再谈判, 那么风险以合同为纽带可 划分为缔约阶段风险和履约阶段风险。合同签订前出现 的风险以及根据以往经验预测未来可能出现的风险为缔 约阶段风险; 履约阶段风险指合同订立后, 项目实施过 程等阶段出现的风险及在缔约阶段残留或变异的风险。 通过对 4 轮 Delphi 法调研结果的统计分析, 结合以往文 献研究成果, 将旧工业建筑再生利用项目风险按缔约与 履约阶段的表现形式划分为 5 大类, 包含 19 小类, 具体 风险因素清单如表 1 所示。

表 1. 旧工业建筑再生利用项目风险要素清单

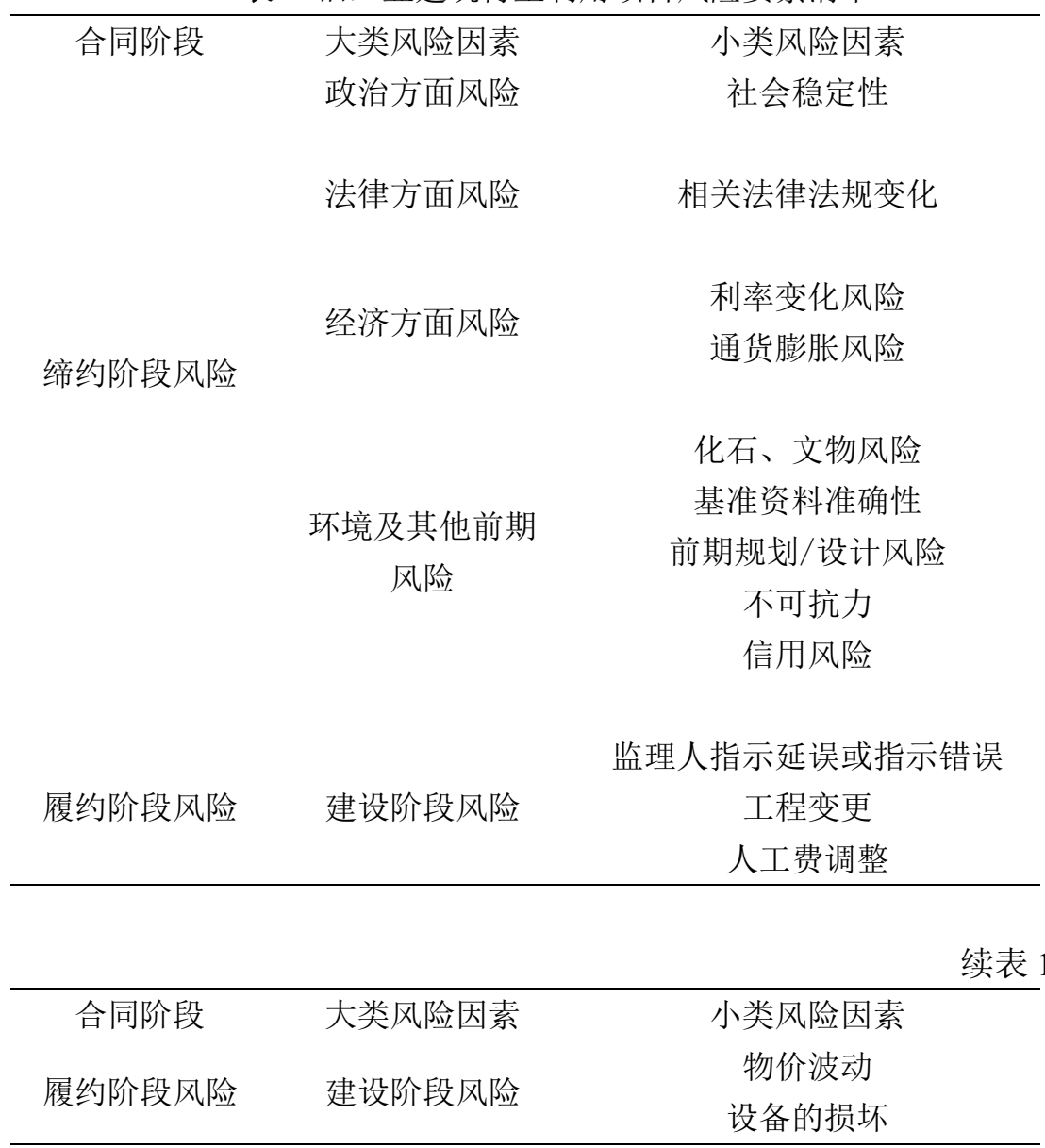




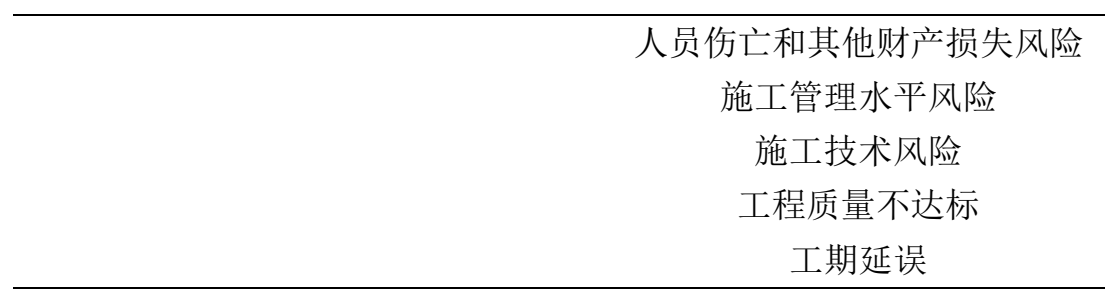

\section{III. 旧工业建筑再生利用项目风险初次分担}

为确保各参与方之间责任归属更加明确, 旧工业建 筑再生利用项目缔约阶段与履约阶段需要充分考虑各风 险被具体分担的情况, 其分担方式包括一方单独承担或 双方共担等。本文首先对项目风险进行初次分担, 界定 出业主方和承包商需单独承担与共担的风险, 其次再确 定共担风险的最优分配比例。

\section{1 风险初次分担多层次指标体系构建}

形成合理的多层次指标体系是风险准确分担的前提, 本文为保证评判的有效性与科学性, 咨询了 10 位旧工业 建筑再生利用项目领域的相关专家, 其中 6 位是具有相 关资质的旧工业建筑再生利用建设技术及管理负责人, 4 位是从事旧工业建筑再生利用研究的高校教授, 选择以 上专家是是因为技术管理贯穿于整个施工过程, 负责人 对旧工业建筑再生利用工程施工技术要求、质量标准等 最为熟悉, 而大学教授研究了大量成功工程项目, 具有 较为丰富的理论基础, 选取的 10 位专家包含在 Delphi 法识别风险时所邀请的 20 位专家内, 结合以往研究文献 [7] 及旧工业建筑再生利用项目特点, 最终形成基于参与 方风险满意度的多层次指标体系，如图 1 所示。

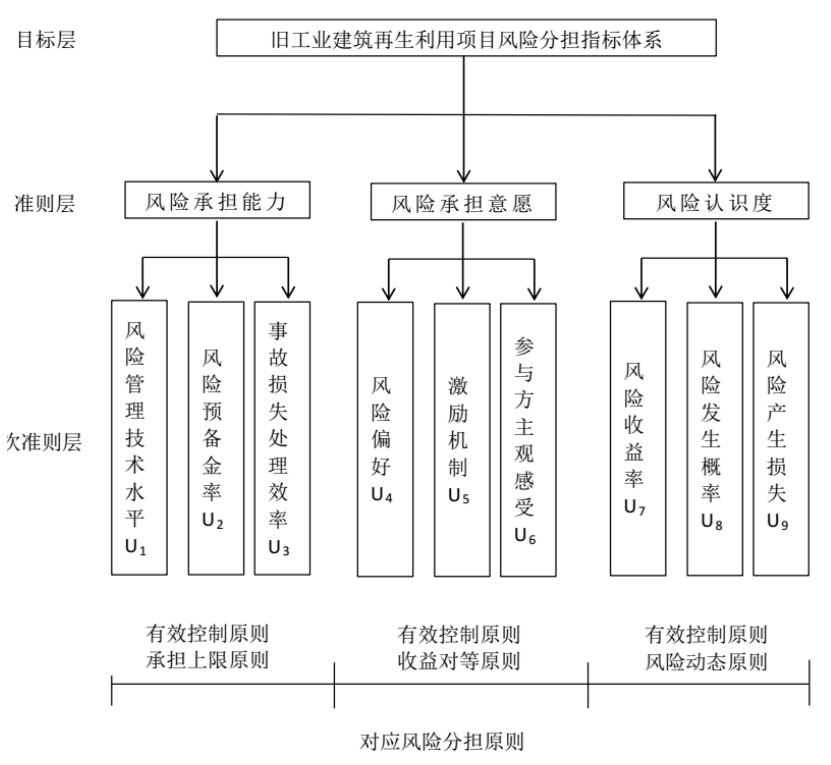

图 1 基于参与方满意度的风险分担指标体系

\section{2 基于 TOPSIS 模型的风险初次分担}

TOPSIS 法是 HWANG, C. L. 和 YoON,K 于 1981 年首 次提出的一种新的多目标决策优选方法。它对数据资料
无太高要求, 可以将复杂的多维空间问题折算到一维空 间进行解决, 凭借其优势已成功应用于众多领域。考虑 到旧工业建筑再生利用项目在国内的研究面不广, 基础 数据库相对薄弱, 且参与方间的风险合理分担属于多目 标决策问题, 故借助 TOPSIS 法构建风险的初次分担模 型。

\subsection{1 风险分担指标体系权重确定}

本文采用基于组合数的有序加权平均 C-OWA(Continuous, Orderd Weighted Averaging)算子对指 标进行赋权, 该算子将专家所赋权重与组合数相结合, 实现对评价指标的科学加权 ${ }^{[8]}$ 。具体, 计算步骤如下所示:

(1)邀请确定风险分担指标体系时的 10 位相关领域 专家对风险分担指标的重要程度进行评判, 采用十分制 打分法，构成指标的原始集合用 $\mathrm{A}=\{\mathrm{a} 1 ， \mathrm{a} 2 ， \cdots, \mathrm{a} 10\}$ 表示, 将原始数据按从大到小顺序进行排列后从 0 开始 编号, 得到新的数据集合即 $\mathrm{B}=\{\mathrm{b} 1, \mathrm{~b} 2, \cdots, \mathrm{b} 10\}$ 。

(2) 由组合数 $C_{h-1}^{n}$ 决定集合 $\mathrm{B}$ 中各数据的权重, 用 $\varphi_{\mathrm{n}+1}$ 表示:

$\varphi_{\mathrm{n}+1}=\frac{\mathrm{C}_{\mathrm{h}-1}^{\mathrm{n}}}{\sum_{\mathrm{n}=0}^{\mathrm{h}} \mathrm{C}_{\mathrm{h}-1}^{\mathrm{n}}}=\frac{\mathrm{C}_{\mathrm{h}-1}^{\mathrm{n}}}{2^{\mathrm{h}-1}}, \mathrm{n}=0,1,2, \cdots, \mathrm{h}-1$

式中: $\sum_{\mathrm{n}=0}^{\mathrm{h}-1} \varphi_{\mathrm{n}+1}=1, \mathrm{~h}$ 为专家数。

(3)用上述数据权重 $\varphi_{\mathrm{n}+1}$ 对数据集合 B 加权, 得评价 指标的绝对权重, 用 $\gamma_{\mathrm{m}}$ 表示:

$\gamma_{\mathrm{m}}=\sum_{\mathrm{n}=0}^{\mathrm{h}-1} \mathrm{~b}_{\mathrm{n}} \varphi_{\mathrm{n}+1}, \mathrm{~m}=1,2, \cdots, \mathrm{z}$

式中: $m$ 为评价指标个数。

(4)计算评价指标的相对权重, 用 $\mathrm{W}_{\mathrm{m}}$ 表示:

$$
\mathrm{W}_{\mathrm{m}}=\frac{\gamma_{\mathrm{m}}}{\sum_{\mathrm{m}=1}^{\mathrm{z}} \gamma_{\mathrm{m}}}
$$

\subsubsection{TOPSIS 模型的具体步骤}

TOPSIS 模型的基本思路是以靠近或远离正负理想 解作为评价各对象的依据, 将各指标因素与分担方之间 贴近度大小进行排序, 并根据排序结果选出风险因素的 最优承担者, 具体计算步骤如下:

(1)指标体系量化及规范化处理

首先设定 $\mathrm{q}$ 个参与方, $\mathrm{m}$ 个评价指标, 针对难以量 化的指标邀请 20 位相关领域专家按 1 9 标度法进行评 判, 这 20 位专家均来自 Delphi 法时确定指标因素的专 家小组成员, 其次为减少专家主观因素的影响, 取专家 组成员打分的平均值作为指标因素的最终评价值, 得指 标因素值 $x_{i j}(1 \leqslant i \leqslant q, 1 \leqslant j \leqslant m)$, 然后得决策矩阵为 $X=$ 
$\left(\mathrm{x}_{\mathrm{ij}}\right)_{\mathrm{q} \times \mathrm{m}}$ 。

使用向量归一化法对决策矩阵 $\mathrm{X}$ 进行规范化处理, 得标准化矩阵 $Y=\left(y_{i j}\right)_{q \times m}$

$$
y_{i j}=\frac{x_{i j}}{\sqrt{\sum_{i=1}^{q} x_{i j}^{2}}}, \quad i=1,2, \cdots, q, j=1,2, \cdots, m
$$

(2)在确定各指标权重基础上, 用下式计算加权标准 化矩阵:

$$
P=\left(p_{i j}\right)_{q \times m}=\left(w_{j} y_{i j}\right)_{q \times m}=\left[\begin{array}{cccc}
p_{1}(1) & p_{1}(2) & \cdots & p_{1}(m) \\
p_{2}(1) & p_{2}(2) & \cdots & p_{2}(m) \\
\vdots & \vdots & & \vdots \\
p_{q}(1) & p_{q}(2) & \cdots & p_{q}(m)
\end{array}\right]
$$

(3)确定各评价指标因素的正理想解 $\mathrm{P}_{0}^{+}$与负理想解 $\mathrm{P}_{0}^{-}$

$$
\mathrm{P}_{0}^{+}=\left\{\left(\max _{1 \leq \mathrm{i} \leq \mathrm{q}} \mathrm{p}_{\mathrm{i}}(\mathrm{j}) / \mathrm{j} \in \mathrm{J}^{+}, \min _{1 \leq \mathrm{i} \leq \mathrm{q}} \mathrm{p}_{\mathrm{i}}(\mathrm{j}) / \mathrm{j} \in \mathrm{J}^{-}\right)\right\}=\left(\mathrm{p}_{0}^{+}(1),\right.
$$

$\left.\mathrm{p}_{0}^{+}(2), \quad \ldots, \mathrm{p}_{0}^{+}(\mathrm{j}), \quad \ldots, \mathrm{p}_{0}^{+}(\mathrm{m})\right)$

$$
\mathrm{P}_{0}^{-}=\left\{\left(\min _{1 \leq \mathrm{i} \leq \mathrm{q}} \mathrm{p}_{\mathrm{i}}(\mathrm{j}) / \mathrm{j} \in \mathrm{J}^{+}, \max _{1 \leq \mathrm{i} \leq \mathrm{q}} \mathrm{p}_{\mathrm{i}}(\mathrm{j}) / \mathrm{j} \in \mathrm{J}^{-}\right)\right\}=\left(\mathrm{p}_{0}^{-}(1),\right.
$$

$\left.\mathrm{p}_{0}^{-}(2), \quad \ldots, \mathrm{p}_{0}^{-}(\mathrm{j}), \quad \ldots, \mathrm{p}_{0}^{-}(\mathrm{m})\right)$

式中： J+表示数值越大越优的指标集合, 具有效益 属性; $\mathrm{J}^{-}$表示数值越大越劣的指标集合, 具有成本属性。

(4) 确定各指标因素到正负理想解 $\mathrm{d}_{\mathrm{i}}^{+}$与负理想解 $\mathrm{d}_{\mathrm{i}}^{-}$ 的距离

$$
\begin{aligned}
& d_{i}^{+}=\sqrt{\sum_{j=1}^{m}\left(p_{i}(j)-p_{0}^{+}(j)\right)^{2}}, i=1,2, \cdots, q \\
& d_{i}^{-}=\sqrt{\sum_{j=1}^{m}\left(p_{i}(j)-p_{0}^{-}(j)\right)^{2}}, i=1,2, \cdots, q
\end{aligned}
$$

(5)确定相对贴近度值 $\mathrm{F}_{\mathrm{i}}^{*}$, 并以此值排序选择风险最 优承担者。

$$
\mathrm{F}_{\mathrm{i}}^{*}=\frac{\mathrm{d}_{\mathrm{i}}^{-}}{\mathrm{di}_{\mathrm{i}}^{+}+\mathrm{d}_{\mathrm{i}}^{-}}
$$

\section{IV. 旧工业建筑再生利用项目共担风险比例确定}

对共同承担的风险, 需要确定各参与方的具体风险 分担比例。风险对旧工业建筑再生利用项目的影响可归 纳为成本，而效用理论是用来描述不确定条件下的决策
行为理论 ${ }^{[9]}$, 能够较好地均衡项目的成本与效用问题。工 程建设项目参与方的管理和控制风险的效用函数可以表 示为收益 $\mathrm{V}$ 和成本 $\mathrm{C}$ 的函数，即：

$$
\mathrm{U}=\mathrm{U}(\mathrm{V}, \mathrm{C})
$$

则工程建设项目的风险分担的模型可表示为:

$$
\left\{\begin{array}{l}
\max \left\{\mathrm{U}_{1}\left(\mathrm{~V}_{1}, \mathrm{C}_{1}\right)+\mathrm{U}_{2}\left(\mathrm{~V}_{2}, \mathrm{C}_{2}\right)\right\} \\
\operatorname{minC}_{\mathrm{a}}\left(\mathrm{C}_{1}, \mathrm{C}_{2}\right)
\end{array}\right.
$$

式中: $\mathrm{U}_{1} 、 \mathrm{~V}_{1} 、 \mathrm{C}_{1}$ 为承包商的效用函数、承担风险 的收益及承担风险的成本; $U_{2} 、 V_{2} 、 C_{2}$ 为业主方的效用 函数、承担风险的收益及承担风险的成本; $\mathrm{C}_{\mathrm{a}}$ 为风险控 制与管理的实际耗用的成本为。

假设风险控制与管理的预估总成本为 $C_{e}, K$ 为工程 建设项目承包商所承担风险成本的比例, $\mathrm{K}$ 的取值范围 为: $0 \leqslant \mathrm{~K} \leqslant 1$; 那么工程建设项目发起方承担的比例为 $1-\mathrm{K}$ 。则:

$$
\left\{\begin{aligned}
C_{e}=C_{e 1}+C_{e 2} ; C_{a}=C_{a 1}+C_{a 2} \\
C_{e 1}=K \times C_{e} ; C_{a 1}=K \times C_{a} \\
\quad C_{e 2}=(1-K) \times C_{e} ; C_{a 2}=(1-K) \times C_{a}
\end{aligned}\right.
$$

旧工业建筑再生利用项目风险的实际成本可用预期 成本的有效性函数表示:

$$
\left\{\begin{aligned}
C_{\mathrm{a} 1} & =\mathrm{M}\left(\mathrm{K}, \mathrm{C}_{\mathrm{e} 1}\right) \\
\mathrm{C}_{\mathrm{a} 2} & =\mathrm{G}\left(1-\mathrm{K}, \mathrm{C}_{\mathrm{e} 2}\right)
\end{aligned}\right.
$$

式中: $M 、 G$ 分别为承包商、业主方对风险控制与 管理的有效性函数。

由(13)、(14)可得:

$$
\mathrm{C}_{\mathrm{a}}=\mathrm{C}_{\mathrm{a} 1}+\mathrm{C}_{\mathrm{a} 2}=\mathrm{M}\left(\mathrm{K}, \mathrm{C}_{\mathrm{e} 1}\right)+\mathrm{G}\left(1-\mathrm{K}, \mathrm{C}_{\mathrm{e} 2}\right)=
$$

$\mathrm{M}\left(\mathrm{K}, \mathrm{K} \times \mathrm{C}_{\mathrm{e}}\right)+\mathrm{G}\left(1-\mathrm{K}, \quad(1-\mathrm{K}) \times \mathrm{C}_{\mathrm{e}}\right)$

$\mathrm{C}_{\mathrm{e}}$ 在签订合同时此值已为已知, 为风险控制与管理 的预估总成本。所以, 风险分担所耗用的实际成本 $\mathrm{C}_{\mathrm{a}}$ 与 业主和承包商之间的风险分担比例密切相关, 是一个关 于 $\mathrm{K}$ 的函数。

在对项目风险有效控制的前提下，工程建设中风险 发生的概率及造成的损失会有所降低, 导致实际成本会 低于预期成本，产生部分风险收益，即：

$$
\left\{\begin{array}{c}
\mathrm{V}_{1}=\mathrm{C}_{\mathrm{e} 1}-\mathrm{C}_{\mathrm{a} 1}=\mathrm{K} \times\left(\mathrm{C}_{\mathrm{e}}-\mathrm{C}_{\mathrm{a}}\right) \\
\mathrm{V}_{2}=\mathrm{C}_{\mathrm{e} 2}-\mathrm{C}_{\mathrm{a} 2}=(1-\mathrm{K}) \times\left(\mathrm{C}_{\mathrm{e}}-\mathrm{C}_{\mathrm{a}}\right)
\end{array}\right.
$$

将公式(13)、(14)、(15)、(16)带入(12)得：

$$
\left\{\begin{array}{r}
\max \left\{\begin{array}{c}
\mathrm{U}_{1}\left(\mathrm{~K} \times\left(\mathrm{C}_{\mathrm{e}}-\mathrm{C}_{\mathrm{a}}\right), \mathrm{K} \times \mathrm{C}_{\mathrm{a}}\right)+ \\
\mathrm{U}_{2}\left((1-\mathrm{K}) \times\left(\mathrm{C}_{\mathrm{e}}-\mathrm{C}_{\mathrm{a}}\right),(1-\mathrm{K}) \times \mathrm{C}_{\mathrm{a}}\right)
\end{array}\right\} \\
\min \left\{\mathrm{M}\left(\mathrm{K}, \mathrm{K} \times \mathrm{C}_{\mathrm{e}}\right), \mathrm{G}\left(1-\mathrm{K},(1-\mathrm{K}) \times \mathrm{C}_{\mathrm{e}}\right)\right\}
\end{array}\right.
$$


但是在工程建设项目的谈判中，承发包双方的谈判 地位并不相等, 所以双方就未必能达到效用函数的最大 化, 所以引入双方在谈判中的权重系数, 构建一个综合 效用目标函数:

$$
\text { f }\left(U_{1}, U_{2}\right)=\omega_{1} U_{1}+\omega_{2} U_{2}
$$

式中: $\omega_{1} 、 \omega_{2}$ 分别为工程建设项目承包商、业主方 在合同谈判中的权重系数, 且 $\omega_{1}+\omega_{2}=1$ 。

业主方和承包商之间进行风险分担谈判的结果是实 现预期效用的最大化, 即: $\operatorname{minC}_{\mathrm{a}}\left(\mathrm{C}_{1}, \mathrm{C}_{2}\right)=\overline{\mathrm{EC}}$

进一步将综合效用目标函数的优化模型转化为:

$$
\begin{gathered}
\operatorname{maxf}\left(\mathrm{U}_{1}, \mathrm{U}_{2}\right)=\max \left\{\omega _ { 1 } \mathrm { U } _ { 1 } \left(\mathrm{K} \times\left(\mathrm{C}_{\mathrm{e}}-\mathrm{C}_{\mathrm{a}}\right), \mathrm{K} \times\right.\right. \\
\left.\mathrm{C}_{\mathrm{a}}\right)+\omega_{2} \mathrm{U}_{2}\left((1-\mathrm{K}) \times\left(\mathrm{C}_{\mathrm{e}}-\mathrm{C}_{\mathrm{a}}\right),(1-\mathrm{K}) \times\right.
\end{gathered}
$$

约束条件: $\mathrm{C}_{\mathrm{a}}=\overline{\mathrm{EC}}$

$\left.\left.\mathrm{C}_{\mathrm{a}}\right)\right\}$

对 $\mathrm{f}\left(\mathrm{U}_{1}, \mathrm{U}_{2}\right)$ 中工程建设项目承包商所承担风险成 本的比例 $\mathrm{K}$ 求偏导, 有:

$$
\begin{aligned}
& \frac{\partial \mathrm{f}}{\partial \mathrm{k}}=\omega_{1} \times\left[\left(\mathrm{C}_{\mathrm{e}}-\mathrm{C}_{\mathrm{a}}\right) \frac{\partial \mathrm{U}_{1}}{\partial \mathrm{V}_{1}}-\mathrm{K} \frac{\partial \mathrm{U}_{1}}{\partial \mathrm{V}_{1}} \frac{\partial \mathrm{C}_{\mathrm{a}}}{\partial \mathrm{K}}+\mathrm{C}_{\mathrm{a}} \frac{\partial \mathrm{U}_{1}}{\partial \mathrm{C}_{1}}+\right. \\
& \left.\mathrm{K} \frac{\partial \mathrm{U}_{1}}{\partial \mathrm{C}_{1}} \frac{\partial \mathrm{C}_{\mathrm{a}}}{\partial \mathrm{K}}\right]+\omega_{2} \times\left[-\left(\mathrm{C}_{\mathrm{e}}-\mathrm{C}_{\mathrm{a}}\right) \frac{\partial \mathrm{U}_{2}}{\partial \mathrm{V}_{2}}-(1-\mathrm{K}) \frac{\partial \mathrm{U}_{2}}{\partial \mathrm{V}_{2}} \frac{\partial \mathrm{C}_{\mathrm{a}}}{\partial \mathrm{K}}+\right. \\
& \left.\mathrm{C}_{\mathrm{a}} \frac{\partial \mathrm{U}_{2}}{\partial \mathrm{C}_{2}}+(1-\mathrm{K}) \frac{\partial \mathrm{U}_{2}}{\partial \mathrm{C}_{2}} \frac{\partial \mathrm{C}_{\mathrm{a}}}{\partial \mathrm{K}}\right]=0
\end{aligned}
$$

由上式可以求出工程建设项目承包商最优风险分担 比例 $K$ 为关于 $\omega_{1}$ 和 $\omega_{2}$ 的函数, 令 $\omega_{1}=\omega$, 则 $\omega_{2}=1-\omega$, 得:

$\mathrm{K}=\eta(\omega)$

运用拉格朗日定理求最优解可得:

$$
\begin{aligned}
\mathrm{L}= & \mathrm{f}\left(\mathrm{U}_{1}, \mathrm{U}_{2}\right)+\lambda\left(\mathrm{C}_{\mathrm{a}}-\overline{\mathrm{EC}}\right) \\
& =\omega \mathrm{U}_{1}\left(\mathrm{~K} \times\left(\mathrm{C}_{\mathrm{e}}-\mathrm{C}_{\mathrm{a}}\right), \mathrm{K} \times \mathrm{C}_{\mathrm{a}}\right) \\
& +(1-\omega) \mathrm{U}_{2}((1-\mathrm{K}) \\
& \left.\times\left(\mathrm{C}_{\mathrm{e}}-\mathrm{C}_{\mathrm{a}}\right),(1-\mathrm{K}) \times \mathrm{C}_{\mathrm{a}}\right) \\
& +\lambda\left(\mathrm{C}_{\mathrm{a}}-\overline{\mathrm{EC}}\right)
\end{aligned}
$$

令: $\frac{\partial \mathrm{L}}{\partial \omega}=0, \frac{\partial \mathrm{L}}{\partial \lambda}=0$ 可得:

$\frac{\partial \mathrm{L}}{\partial \omega}=\mathrm{U}_{1}\left[\mathrm{~K} \times\left(\mathrm{C}_{\mathrm{e}}-\mathrm{C}_{\mathrm{a}}\right), \quad \mathrm{K} \times \mathrm{C}_{\mathrm{a}}\right]+\omega \times\left[\left(\mathrm{C}_{\mathrm{e}}-\right.\right.$
$\left.\left.\mathrm{C}_{\mathrm{a}}\right) \frac{\partial \mathrm{U}_{1}}{\partial \mathrm{V}_{1}} \eta^{\prime}(\omega)+\mathrm{C}_{\mathrm{a}} \frac{\partial \mathrm{U}_{1}}{\partial \mathrm{C}_{1}} \eta^{\prime}(\omega)\right]-(1-\omega) \times\left[\left(\mathrm{C}_{\mathrm{e}}-\right.\right.$

$\left.\left.\mathrm{C}_{\mathrm{a}}\right) \frac{\partial \mathrm{U}_{2}}{\partial \mathrm{V}_{2}} \eta^{\prime}(\omega)+\mathrm{C}_{\mathrm{a}} \frac{\partial \mathrm{U}_{2}}{\partial \mathrm{C}_{2}} \eta^{\prime}(\omega)\right]-\mathrm{U}_{2}\left[(1-\mathrm{K}) \times\left(\mathrm{C}_{\mathrm{e}}-\mathrm{C}_{\mathrm{a}}\right)\right.$,

$\left.(1-\mathrm{K}) \times \mathrm{C}_{\mathrm{a}}\right]=0$

$$
\frac{\partial \mathrm{L}}{\partial \lambda}=\mathrm{C}_{\mathrm{a}}-\overline{\mathrm{EC}}=0
$$

由式(24)解出:

$$
\mathrm{C}_{\mathrm{a}}=\mu(\omega)
$$

即 $C_{a}$ 为关于 $\omega$ 的函数, 将(25)带入(15)得:

$$
\mathrm{K}=\beta(\omega)
$$

由式(27)可知: $K$ 为关于 $\omega$ 的一个函数, 确定风险双 方谈判中的权重系数 $\omega$ 后可以根据以上模型计算风险合 理分担的比例系数，确定双方共担风险的具体分配比例。

\section{V.案例分析}

采用位于西安市东郊新城区的陕西某钢厂再生利用 项目验证风险分担模型的合理性。该钢厂在上世纪 50 年 代末建成, 为响应国家可持续发展要求及绿色发展理念, 拟将其部分老厂房改造为创意产业园。园区总占地面积 约 50 亩, 原有建筑面积约 1.5 万 $\mathrm{m}^{2}$, 再生利用后总建筑 面积约 4 万 $\mathrm{m}^{2}$, 将园区内保存的 8 栋原有厂房进行功能 分区, 划分成 11 栋新型办公楼, 并新建一栋 9\#楼。构成 由 “多功能活动厅、创意办公区、创意设计展示区、创 意广场、商务休闲区、生活休闲区” 六部分组成的低密 度、花园式办公为主导的创意工作、生活、交流学习为 一体的西安时尚基地，项目总投资 3147.86 万元。

本文选择业主方和承包商作为主要研究对象, 运用 TOPSIS-UT 模型合理分担该项目风险。

\section{1 基于 TOPSIS 模型的风险初次分担}

旧工业建筑再生利用项目在缔约与履约阶段的风险 因素如表 1 所示, 本文在风险初次分担阶段以 “基准资 料准确性”为例进行计算。

\subsection{1 风险分担指标体系权重确定}

如图 1 所示, 构建基于参与方满意度的风险分担指 标体系。邀请 10 位旧工业建筑再生利用项目领域专家按 指标重要程度以 10 分制进行评判, 具体打分情况如表 2 所示。

表 2. 专家打分结果

\begin{tabular}{ccccccccccc}
\hline 风险分 & \multicolumn{10}{c}{ 专家 } \\
担指标 & $(1)$ & $(2)$ & $(3)$ & $(4)$ & $(5)$ & $(6)$ & $(7)$ & $(8)$ & 9 & (10) \\
$\mathrm{U}_{1}$ & 8.5 & 9 & 8 & 7.5 & 8.5 & 9 & 8.5 & 8 & 9 & 9 \\
$\mathrm{U}_{2}$ & 7 & 7.5 & 7.5 & 6 & 6 & 7 & 6.5 & 6.5 & 5 & 6 \\
\hline
\end{tabular}




\begin{tabular}{ccccccccccc}
\hline $\mathrm{U}_{3}$ & 9 & 8.5 & 8.5 & 8 & 7.5 & 8 & 7 & 8.5 & 8.5 & 8 \\
$\mathrm{U}_{4}$ & 5 & 6 & 6 & 6.5 & 6 & 5 & 5 & 5.5 & 4.5 & 6 \\
$\mathrm{U}_{5}$ & 7 & 6.5 & 6.5 & 7 & 5.5 & 5 & 6.5 & 6 & 6 & 6.5 \\
\hline
\end{tabular}

续表 2

\begin{tabular}{ccccccccccc} 
风险分 & \multicolumn{10}{c}{ 专家 } \\
担指标 & 1 & 2 & $(3$ & 4 & 5 & 6 & 7 & 8 & 9 & 10 \\
$\mathrm{U}_{6}$ & 5.5 & 6 & 5.5 & 5 & 5.5 & 5 & 6 & 4 & 5.5 & 5.5 \\
$\mathrm{U}_{7}$ & 6.5 & 8 & 7 & 8.5 & 7 & 6 & 7 & 6.5 & 6.5 & 7 \\
$\mathrm{U}_{8}$ & 6 & 7 & 6.5 & 8 & 7 & 6 & 6 & 7 & 6 & 6.5 \\
$\mathrm{U}_{9}$ & 7 & 8 & 9 & 7.5 & 6.5 & 7.5 & 7.5 & 7 & 8 & 6
\end{tabular}

以指标 “风险管理技术水平 $U_{1}$ ” 为例进行计算, 将 $\quad \mathrm{W}=(0.1425,0.0942,0.1276,0.0921,0.1076,0.0907,0.1144,0.1$ 10 位专家打分的原始数据从大到小顺序进行排列, 得到 新的数据集合 $\mathrm{B}=\{9,9,9,9,8.5,8.5,8.5,8,8$, 7.5 \}; 由式(1)得到各数据的权重:

$\varphi=(0.0019,0.0176,0.0703,0.1641,0.2461,0.2461$, $0.1641,0.0703,0.0176,0.0019)$

运用式 (2) 得评价指标绝对权重:

$\gamma_{1}=(9,9,9,9,8.5,8.5,8.5,8,8,7.5) \cdot(0.0019$, $0.0176,0.0703,0.1641 。 0.2461,0.2461,0.1641,0.0703$, $0.0176,0.0019)^{\mathrm{T}}=8.5811$

同理得 $\gamma_{2}=5.6733, \gamma_{3}=7.6867, \gamma_{4}=5.5446$, $\gamma_{5}=6.4784, \gamma_{6}=6.4784, \gamma_{7}=6.8926, \gamma_{8}=6.5019$, $\gamma_{9}=7.4053$ 。最后运用式（3）得各指标的绝对权重: $080,0.1229)$

\subsubsection{TOPSIS 模型的具体步骤}

(1)指标体系量化及规范化处理

针对 “基准资料准确性” 风险因素, 邀请 20 位相关 领域专家按 1 9 标度法对其进行评判, 取 20 位专家打分 的平均值作为指标评价值, 并利用式(4)对数据进行规范 化处理, 得标准化矩阵:

$$
\begin{gathered}
\mathrm{Y}=\left[\begin{array}{lllll}
0.4997 & 0.5459 & 0.5235 & 0.4921 & 0.5362 \\
0.8662 & 0.8377 & 0.8520 & 0.8706 & 0.8441 \\
0.4375 & 0.4561 & 0.5963 & 0.4576 \\
0.8992 & 0.8899 & 0.8027 & 0.8891
\end{array}\right]
\end{gathered}
$$

即承包商与业主方的标准值如表 3 所示:

表 3. 参与方评价指标标准值

\begin{tabular}{cccccccccc}
\hline 参与方 & $\mathrm{U}_{1}$ & $\mathrm{U}_{2}$ & $\mathrm{U}_{3}$ & $\mathrm{U}_{4}$ & $\mathrm{U}_{5}$ & $\mathrm{U}_{6}$ & $\mathrm{U}_{7}$ & $\mathrm{U}_{8}$ & $\mathrm{U}_{9}$ \\
承包商 & 0.4997 & 0.5459 & 0.5235 & 0.4921 & 0.5362 & 0.4375 & 0.4561 & 0.5963 & 0.4576 \\
业主方 & 0.8662 & 0.8377 & 0.8520 & 0.8706 & 0.8441 & 0.8992 & 0.8899 & 0.8027 & 0.8891 \\
\hline
\end{tabular}

(2)计算加权标准化矩阵

确定各指标权重后, 运用式(5)得加权标准化矩阵:

$P=\left(p_{i j}\right)_{2 \times 9}=\left(w_{j} y_{i j}\right)_{2 \times 9}=\left[\begin{array}{lll}0.0712 & 0.0514 & 0.0668 \\ 0.1234 & 0.0781 & 0.1087\end{array}\right.$

$\left.\begin{array}{llllll}0.0453 & 0.0577 & 0.0397 & 0.0522 & 0.0644 & 0.1093 \\ 0.0802 & 0.0908 & 0.0815 & 0.1018 & 0.0867 & 0.1093\end{array}\right]$

(3) 确定正理想解 $\mathrm{P}_{0}^{+}$与负理想解 $\mathrm{P}_{0}^{-}$

根据 $J^{+}$与 $J^{-}$的选择原则, $\mathrm{U}_{1}, \mathrm{U}_{2}, \mathrm{U}_{3}, \mathrm{U}_{4}, \mathrm{U}_{5}, \mathrm{U}_{6}$, $\mathrm{U}_{7}$ 属于 $\mathrm{J}^{+}$集合; $\mathrm{U}_{8}, \mathrm{U}_{9}$ 属于 $\mathrm{J}^{-}$集合, 利用式(6), (7)求得 正理想解 $\mathrm{P}_{0}^{+}$及负理想解 $\mathrm{P}_{0}^{-}$为:

$$
\begin{aligned}
& \mathrm{P}_{0}^{+}=\left(\begin{array}{llll}
0.1234, & 0.0781, & 0.1087, & 0.0802,0.0908
\end{array}\right. \\
& 0.0815,0.1018,0.0644,0.0562) \\
& \mathrm{P}_{0}^{-}=\left(\begin{array}{lllll}
0.0712, & 0.0514, & 0.0668, & 0.0453, & 0.0577
\end{array}\right. \\
& 0.0397,0.0522,0.0867,0.1093)
\end{aligned}
$$$$
\text { 表 4. 陕西某钢厂再生利用为创意产业园项目风险初次分担结果 }
$$

(4)确定指标因素到正负理想解 $\mathrm{d}_{\mathrm{i}}^{+}$与负理想解 $\mathrm{d}_{\mathrm{i}}^{-}$的 距离

利用公式(8), (9)得:

$\mathrm{d}_{\mathrm{i}}^{+}=(0.1191,0.0576) \quad \mathrm{d}_{\mathrm{i}}^{-}=\left(\begin{array}{ll}0.0576,0.1191\end{array}\right)$

(5)确定相对贴近度值 $F_{i}^{*}$

由式(10)得相对贴近度:

$$
\mathrm{F}_{\mathrm{i}}^{*}=(0.3260,0.6740)
$$

由以上结果可知, 业主方相对贴近度大, 表示与理 想风险分担方案最接近, 是最适合承担 “基准资料准确 性” 风险的参与方, 因此, 本文构建的 TOPSIS 初次风 险分担模型的合理性。通过咨询专家及参考文献 ${ }^{[10]}$ 知, 当针对某一风险的不同参与方求得相对贴近度数值接近 且差值在 0.05 范围内时共同承担该风险。由此可计算出 其他风险的最佳承担方, 如表 4 所示。 
相关法律法规变化、化石文物风险、基准资料准确 性、前期规划/设计风险、监理人指示延误或指示错

业主方

误、工程变更、人工费调整

风险承担类型险类别 风险承担主体

续表 4

社会稳定性、利率变化风险、通货膨胀风险、不可

共同承担风险抗力、信用风险、物价波动、人员伤亡和其他财产承包商、业主方

损失风险、工程质量不达标、工期延误

\section{2 基于效用理论确定共担风险比例}

陕西某钢厂再生利用为创意产业园项目需要参与方 共同承担的风险如表 4 所示，本文以需要承包商和业主 方共同承担的不可抗力风险为例计算具体分担比例。承 包商预估的预期风险成本为 $C_{e 1}=300$ 万元，业主方预估 的预期风险成本为 $\mathrm{C}_{\mathrm{e} 2}=500$ 万元，参考文献 ${ }^{[11]}$ 假设双方 谈判的权重系数比为 $\omega_{1}: \omega_{2}=2: 8$, 并且根据业主方和 承包商所收集的风险管理经验数据, 采用直接提问法和 对比提问法 ${ }^{[12]}$ 及 L-A 效用函数拟合法 ${ }^{[13]}$ 可得出承包商 与业主方的效用函数为:

$$
\left\{\begin{array}{l}
\mathrm{U}_{1}=-\frac{1}{160} \mathrm{C}_{1}{ }^{2}+\mathrm{V}_{1} \\
\mathrm{U}_{2}=-\frac{1}{480} \mathrm{C}_{2}{ }^{2}+\mathrm{V}_{2}
\end{array}\right.
$$

利用公式(25)可得该风险双方预期成本的平均值为:

$\mathrm{C}_{\mathrm{a}}=\overline{\mathrm{EC}}=\frac{\left(\mathrm{C}_{\mathrm{e} 1}+\mathrm{C}_{\mathrm{e} 2}\right)}{2}=\frac{(300+500)}{2}=400 （$ 万元 $)$

根据本文建立的基于效用理论建立的风险分担模型, 将已知条件 $\mathrm{C}_{\mathrm{e} 1}=300$ 万元; $\mathrm{C}_{\mathrm{e} 2}=500$ 万元; $\mathrm{C}_{\mathrm{a}}=400$ 万 元; $\omega_{1}: \omega_{2}=2: 8$ 带入式(20)可得： $\operatorname{maxf}\left(\mathrm{U}_{1}, \mathrm{U}_{2}\right)=\max \left\{\omega_{1} U_{1}\left(K \times\left(C_{e}-C_{a}\right), K\right.\right.$ $\left.\times C_{a} \quad\right)$

表 5.陕西某钢厂再生利用为创意产业园项目共担风险的最优分配比例

\begin{tabular}{cc}
\hline 风险因素 & 分担比例 \\
社会稳定性 & $0.3807: 0.6193$ \\
利率变化风险 & $0.4908: 0.5092$ \\
通货膨胀风险 & $0.3807: 0.5193$ \\
不可抗力 & $0.4643: 0.5357$ \\
信用风险 & $0.3438: 0.6562$ \\
物价波动 & $0.2500: 0.7500$ \\
人员伤亡和其他财产损失风险 & $0.6545: 0.3455$ \\
工程质量不达标 & $0.6323: 0.3677$ \\
工期延误 & $0.5102: 0.4898$ \\
\hline
\end{tabular}

VI. 结论

本文采用 Delphi 法识别旧工业建筑再生利用项目的 风险因素, 并构建了 TOPSIS-UT 理论的风险分担模型,

$$
\begin{aligned}
& \left.+\omega_{2} U_{2}\left((1-\mathrm{K}) \times\left(C_{e}-C_{a}\right),(1-\mathrm{K}) \times C_{a}\right)\right\} \\
& =\max \left\{0.2 \times U_{1}(K \times(300-400), K \times 400)\right. \\
& \left.\quad+\omega_{2} U_{2}((1-\mathrm{K}) \times(500-400),(1-\mathrm{K}) \times 400)\right\}
\end{aligned}
$$

带入效用函数(28)可得:

$\operatorname{maxf}\left(U_{1}, U_{2}\right)=$

$\max \left\{0.2 \times\left[-\frac{1}{160} \times 400^{2} K^{2}+(300-400) K\right]+\right.$

$\left.0.8 \times\left[-\frac{1}{480} \times 400^{2}(1-K)^{2}+(500-400) \times(1-K)\right]\right\}$ 整理上式得:

$\operatorname{maxf}\left(U_{1}, U_{2}\right)=-\frac{1400}{3} K^{2}+\frac{1300}{3} K-\frac{560}{3}$ $=\max F(\mathrm{~K})$

令 $\frac{d F}{d K}=0$ 时 $F(K)$ 取最大, 可求得 $K=0.4643$

承包商与业主方针对不可抗力风险的最优分担比例 为 0.4643:0.5357, 此时项目的效用只和达到最优。同时, 其他共担风险的最优分担比例计算结果如表 5 所示。 
约理论, 将 Delphi 法识别出的风险因素归为缔约阶段风 险与履约阶段风险两大类，具有科学性。

(2)构建了基于承包商和业主方满意度的风险分担指 标体系, 运用基于组合数的有序加权平均确定指标权重, 避免了数据差值较大造成的不良影响。由计算可知风险 管理技术水平、事故损失处理效率及风险损失等因素的 相对权重较大，在风险分担中影响程度较高。

(3)形成了一套较为完备的风险分担机制，运用 TOPSIS 模型界定出单独承担与共同承担的风险, 利用 UT 理论确定共担风险的最优分担比例, 并利用陕西某钢 厂再生利用项目实例证明在采用该风险分担机制具有一 定合理性与可行性，为旧工业建筑再生利用项目风险分 担提供了参考。

(4)旧工业建筑再生利用项目的风险合理分担有利于 各参与方清楚的了解其应承担的风险及具体比例, 选择 合适的合同类型，明确合同中风险归属，提高合同签订 的效率。

\section{致谢}

本项目受国家自然科学基金面上项目 “绿色节能导向 的旧工业建筑功能转型机理研究”（编号:51678479）资 助。

\section{参考文献}

[1]奘胜军, 李慧民, 路鹏飞。旧工业厂房改造工程施工阶段的风险 评估 $[\mathrm{J}]$. 西安科技大学学报，2008, 28(1):158-162.
[2] 刘文. 老厂房加固改造工程的技术风险分析与评估研究[J]. 地 下空间与工程学报，2012,08(S2):1680-1683.

[3] 高亚男. 单层工业厂房再利用投资决策风险识别及量化研究[D]. 西安:西安建筑科技大学,2013.

[4] 尹志国. 冶金工业项目绿色建造技术与成本风险管理研究[D]. 西安:西安建筑科技大学, 2014

[5] 余晓松. 旧工业建筑改造再利用全过程风险管理研究[D]. 西安： 西安建筑科技大学,2015.

[6] Hart O, Morre J. Property rights and the nature of the firm [J]. Jourrnal of Political Economy, 1990, 98(6): 1119-1158.

[7] 刘江帆. 基于系统动力学的旧工业建筑再利用施工风险管理研 究[D]. 西安建筑科技大学, 2016 .

[8] 宋博, 武瑞娟, 牛发阳. 基于 OWA 与灰色聚类的城市轨道交通 PPP 融资风险评价方法研究[J]. 隧道建设,2017,37(04):435-441.

[9] 赵九茹, 李心广, 李霞. 基于多属性效用理论的群体决策偏好整合 研究[J]. 统计与决策, 2017(21):42-46.

[10] 庄文静, 王利, 姜朋明. 基于熵权-TOPSIS 模型的综合管廊 PPP 项 目风险初步分担研究 [J]. 江苏科技大学学报 (社会科学 版), 2017,17(02):90-97.

[11] 周运先. 工程项目招投标阶段合同风险合理分担研究[D]. 长安 大学, 2013.

[12] 冯利军, 李书全. 效用理论在工程项目风险管理决策中的应用 $[\mathrm{J}]$ 山西建筑,2007(26):14-15.

[13] 安玉英, 李绍文. 效用函数与风险型损益值决策 [J]. 统计研 究, 1986(04):69-76. 